\title{
Fluorspar Resources of Africa
}

G E O L O G I C A L S U R V E Y B U L L E T I N 1487

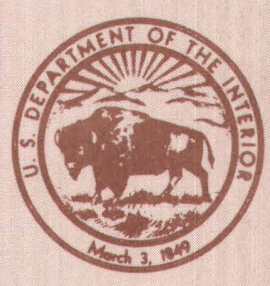





\section{Fluorspar Resources of Africa}

By RALPH E. VAN ALSTINE and PAUL G. SCHRUBEN

G E O L O G I C A L S U R V E Y B U L L E T I N 1487

Geologic setting, production, reserves, outlook for future supply, and relation of fluorspar deposits to rift structures and carbonatites

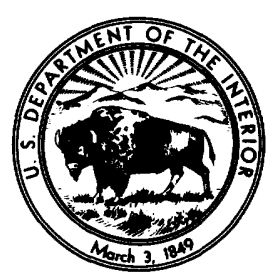




\section{UNITED STATES DEPARTMENT OF THE INTERIOR}

CECIL D. ANDRUS, Secretary

\section{GEOLOGICAL SURVEY}

H. William Menard Director

\section{Library of Congress Cataloging in Publication Data}

Van Alstine, Ralph Erskine, 1914-

Fluorspar resources of Africa.

(Geological Survey bulletin ; 1487)

Bibliography: $p$.

1. Fluorspar-Africa. I. Schruben, Paul G., joint author. II. Title. III. Series: United States.

Geological Survey. Bulletin ; 1487.

QE75.B9 no. $1487 \quad$ [TN948.F6] 557.3'08s [553' 95] 79-20343 


\section{CONTENTS}

Abstract

Introduction

Geologic environments and types of fluorspar deposits

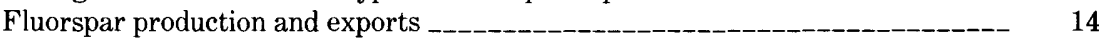

Fluorspar reserves _-_- 17

Outlook for future supply

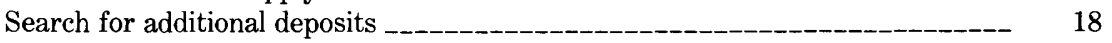

References cited

\section{ILLUSTRATIONS}

Plate 1. Map of Africa showing the location of fluorspar and carbonatite deposits, rifts, and other major faults

FIGURES 1-3. Graphs showing:

1. Comparison of fluorspar production from South Africa with total African fluorspar production, 1918-76

2. African fluorspar exports 1958-73, by area of destination -- 15

3 . Amount of fluorspar imported by the United States, 1920-77

\section{TABLES}

TABLE 1. Fluorite in Africa

2. Selected data on African fluorspar production through 1976

3. African fluorspar reserves

4. Carbonatite localities of Africa 
CONVERSION FACTORS

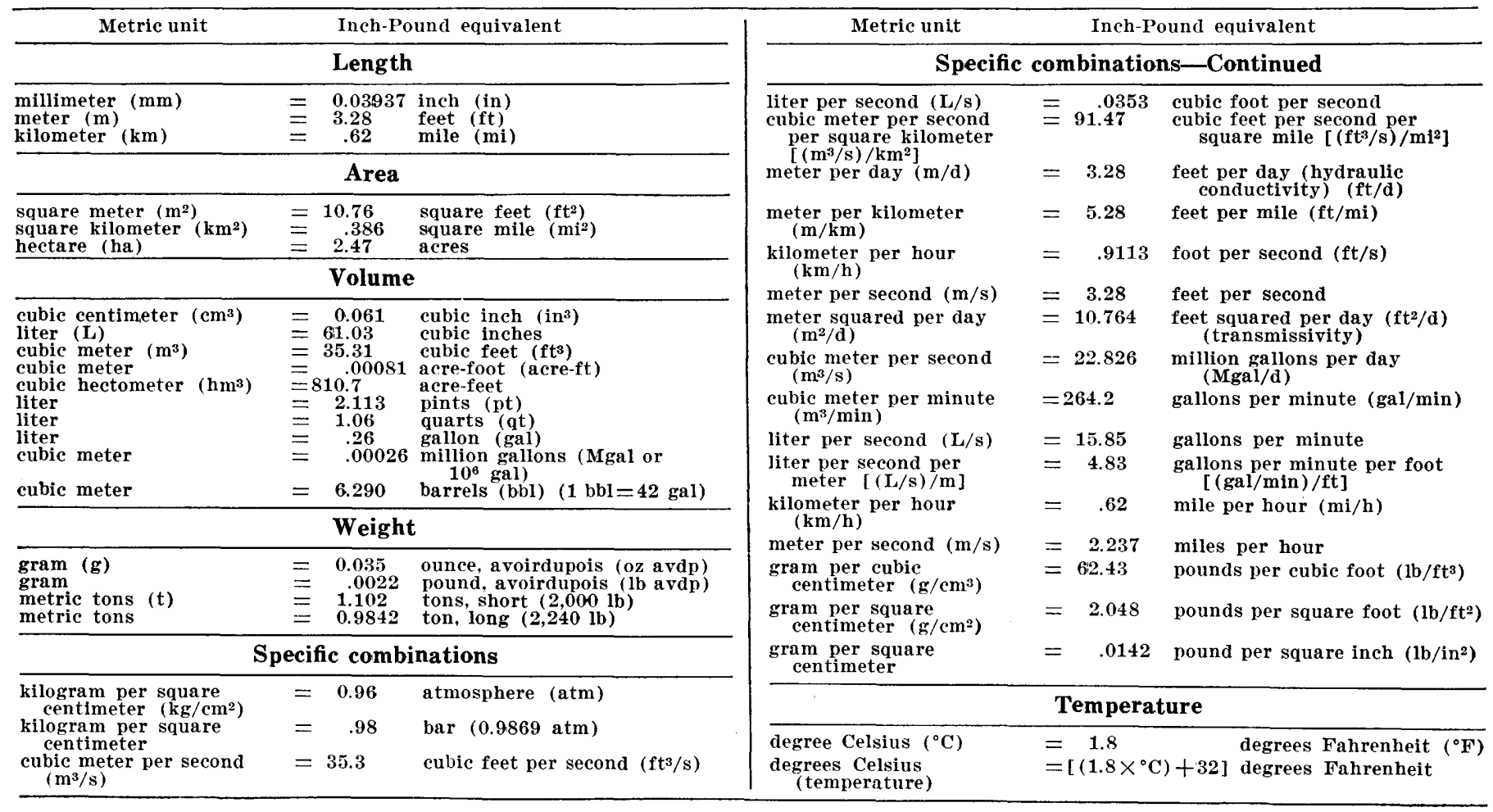




\title{
FLUORSPAR RESOURCES OF AFRICA
}

\author{
By Ralph E. Van Alstine and Paul G. Schruben
}

\begin{abstract}
Africa is becoming one of the world's leading producers and exporters of fluorspar. This compilation is timely because of the increased amounts of African fluorspar imported by the United States. From 1975 through 1977, imports of African fluorspar increased from 4 percent of our total fluorspar imports to 22 percent, or from about 39,000 to about 199,000 metric tons.

Fluorspar ore, as mined in Africa, averages about 22 percent $\mathrm{CaF}_{2}$. It is beneficiated into metallurgical, ceramic, or acid grade for the steel, aluminum, ceramic, and chemical industries.

Fluorspar veins are worked in Egypt, Morocco, and South Africa, but manto fluorspar deposits in carbonate rocks are much more productive in South Africa, Kenya, and Morocco. Pipelike bodies of fluorspar have been worked in South Africa and Namibia. Stockworks of fluorspar in granite are mined extensively in South Africa. Fluoritebearing carbonatites are reported from Angola, Kenya, Morocco, Mozambique, Namibia, South Africa, Tanzania, and Uganda. Fluorite has been reported in 24 of the 115 carbonatites in Africa, but large commercial-grade fluorspar deposits in or associated with carbonatites have been reported only in the Okorusu district of Namibia.

Fluorspar production from Africa through 1976 totals about 3.5 million metric tons. Approximately 88 percent of this total was produced since 1960 , and 80 percent originated in South Africa. In 1976, about 83 percent of African fluorspar production was exported.

Estimated African fluorspar reserves of proved and probable ore total about 192 million metric tons and average 22 percent $\mathrm{CaF}_{2}$. South Africa accounts for about 86 percent of this total and ranks first in world reserves of fluorspar. These reserve estimates for Africa are taken from reports published in 1976 or later.

Africa is able to provide an increasing and a major share of the world's fluorspar supply. South Africa is the only substantial consumer of fluorspar in Africa, and most of the African fluorspar production will continue to be available for export.

Geological conditions are favorable for the discovery of additional large fluorspar deposits. The most promising places to search are in and near carbonatites and alkaline and highly silicic igneous rocks, especially where adjacent rocks consist of readily replaceable limestone or dolomite. The carbonatites and other intrusive bodies are in regions of tension marked by abyssal or subcrustal fractures, rifts, block faults, grabens, and mainly vertical movements. Saline lakes and playas of the rift valleys of Africa may contain fluorine minerals of commercial importance. Fluorine hot springs near the rifts, and also the eroded carbonatites and alkaline and silicic igneous rocks rich in fluorine, contributed fluorine to the basins. Possibly fluorite will be found in Cenozoic tuffaceous lake beds near the rift zones.
\end{abstract}




\section{INTRODUGTION}

Africa is becoming one of the world's leading producers and exporters of fluorspar. In 1977, about 22 percent of the $900,000 \mathrm{t}$ (metric tons) of fluorspar imported into the United States came from Africa. This study is timely because of the increased amounts of African fluorspar exported to the United States, which consumes about 20 percent of the world's annual production of about 5 million metric tons.

In recent years, United States imports of fluorspar from Africa originated chiefly in South Africa (source of 89 percent of our African imports in 1977) and in Kenya, Morocco, and Tunisia. From 1975 through 1977 imports of African fluorspar increased from 4 percent of our total fluorspar imports to 22 percent, or from about 39,000 t to about $199,000 \mathrm{t}$. African fluorspar production in the past 10 years has increased 4.5 times. In view of these facts, the present study was undertaken to appraise the fluorspar resources of Africa, to examine possibilities of increased production and exports, and to consider the effects of such an increase upon the worldwide fluorspar industry.

This report is a compilation of information from published literature and data in the files of the U.S. Geological Survey. The text, tables, and map, where possible, give the name of the deposit or district, location, geologic environment, resources, grade, and sources of data. The references cited are the publications used in preparing this compilation and are those considered most helpful in appraising areas in Africa that contain fluorspar resources.

In industry, fluorspar ore must contain enough of the mineral fluorite $\left(\mathrm{CaF}_{2}\right)$, usually more than 20 percent, to be of commercial interest. Fluorite is 48.7 percent fluorine and 51.3 percent calcium. Its industrial value is due mostly to the fluorine content, and it is the chief source of that element. Fluorspar is used mainly in making hydrofluoric acid, fluorocarbons, and other fluorine chemicals; in manufacturing artificial cryolite and aluminum fluoride for the aluminum industry; as a flux in the steel, iron foundry, and ferroalloy industries; and in the ceramic industry.

Fluorspar, as mined in open pits and underground workings in Africa, averages about 22 percent $\mathrm{CaF}_{2}$. It is beneficiated generally by heavy-media or flotation processes into metallurgical, ceramic, or acid grade. Metallurgical-grade fluorspar, chiefly for the steel industry, contains at least 60 effective percent $\mathrm{CaF}_{2}$ (effective percentage of $\mathrm{CaF}_{2}$ is determined by multiplying the silica percentage in the analysis by 2.5 and subtracting this number from the $\mathrm{CaF}_{2}$ percentage in the analysis). Much metallurgical-grade fluorspar now comes in the form of pellets or briquets because of their convenience and controlled physical properties and chemical composition. Ceramic grades of fluorspar, used for 
making pottery, glass, fiberglass, opal glass, and enamels, contain 85-96 percent $\mathrm{CaF}_{2}$. Acid-grade fluorspar, used by the chemical and aluminum industries, contains at least 97 percent $\mathrm{CaF}_{2}$.

The price of acid-grade filter cake produced by flotation in South Africa was quoted at $\$ 97-\$ 102$ a short ton (Engineering and Mining Journal, 1978); however, acid-grade fluorspar exported in 1976 averaged about 57 rands (\$66) a metric ton (Gössling, 1978, p. 34), the rand being equivalent to $\$ 1.15$, (U.S. dollars) in 1978 . In the United States, the price for acid-grade and ceramic-grade fluorspar ranges from $\$ 95$ to $\$ 115$ a short ton, and that for the metallurgical-grade pellets or briquets, 70 percent effective $\mathrm{CaF}_{2}$, ranges from $\$ 83$ to $\$ 91$ a short ton (Engineering and Mining Journal, 1978).

\section{GEOLOGIC ENVIRONMENTS AND TYPES OF FLUORSPAR DEPOSITS}

Fluorite is a fairly soft (hardness of 4 on the Mohs scale) and moderately heavy ( $\mathrm{sp} \mathrm{gr}, 3.1$ ) isometric mineral that is generally associated with quartz, chalcedony, calcite, or barite; sphalerite and galena are the most common sulfide minerals in fluorspar deposits. Fluorite occurs in a variety of colors, and ranges from large crystals having cubic, octahedral, or dodecahedral forms to fine-grained material having no crystal faces. The fine-grained material may have earthy, botryoidal, reniform, mammillary, and stalactitic structures. Fluorite in tuffaceous lake beds of Western United States is so fine grained that it can be distinguished only with an electron microscope or $\mathrm{X}$-ray. This type of material in a deposit near Rome, Italy, (Spada, 1969 ) is about to be worked commercially.

Fluorspar occurs throughout the world in a great variety of geologic environments and types of deposits. Epigenetic fluorspar deposits commonly coincide with regions of low gravity and zones of high heat flow, igneous activity, and hydrothermal effects (Shawe and others, 1976). The tectonic environment consists chiefly of tensional and deep-seated normal fault zones. The most common types of fluorspar deposits in the world are veins; mantos (bedded layers, stratabound, or stratiform); pipelike bodies (including mineralized diatremes and cryptovolcanic structures) and stockworks; contact zones and greisens; pegmatites and carbonatites; disseminated deposits in granites, syenites, volcanic rocks, and tuffaceous lake beds; and residual deposits derived from the weathering of any of the above types. Highly silicic and alkalic igneous rocks are common associates of fluorspar deposits, but any type of rock may be the host rock for the actual deposit; limestone and dolomite are most favorable. At some localities limestone or dolomite has been strongly fractured, veined, or made cavernous by ground water, and 
ore solutions have deposited fluorite in the open spaces or as replacements of the wall rocks.

Most large concentrations of fluorspar are associated with continental rifts and lineaments throughout the world (Van Alstine, 1976). These structures and fluorspar deposits are of various geologic ages but commonly are of Cenozoic age. Evidently large volumes of fluorine leaked upward from the lower crust or mantle along the rifts and formed the deposits. In Africa fluorspar deposits, ranging in age from Precambrian through Tertiary, are along or near the rift systems, from north to south, in Egypt, Somalia, Uganda, Kenya, Tanzania, Malawi, Mozambique, and South Africa (pl. 1).

Plate 1 shows 76 fluorite localities in 19 countries. Many of these deposits are associated with carbonatites, alkaline intrusive and extrusive rocks, and highly silicic igneous rocks. In South Africa a rift zone projects south from the Great Dyke of Rhodesia, past a swarm of satellite dikes in the Limpopo Belt (Jones and others, 1975), and to the Bushveld Complex. An east-trending arcuate fracture zone connects the Bushveld Complex centers of intrusion (Verwoerd, 1967, p. 282 and fig. 19). Near the intersection of these two Precambrian structures are many intrusions of highly silicic rocks, alkaline rocks, and carbonatites and what may be the largest concentration of fluorspar in the world. The fluorspar mineralization is also considered to be of Precambrian age and related to a late phase of the Bushveld granite. Similarly, fluorite is associated with alkaline rocks, carbonatites, and northtrending or northeast-trending young rifts in eastern and central Africa, in Namibia (South West Africa), and in Angola and is associated with major fault zones in the Atlas Mountains of Morocco and Tunisia.

As shown in table 1, all types of fluorspar deposits described in the preceding paragraphs are in Africa. Table 1 gives selected information on 76 fluorite localities in 19 countries. At present, fluorspar veins actively being mined are in Egypt, Morocco, and South Africa. Mantos or bedded layers of fluorspar in carbonate rocks form large deposits in Kenya, Morocco, and the Marico district of South Africa. Pipelike bodies of fluorspar are exemplified by deposits in the Marico and Kromdraai districts of South Africa and in the Garub deposit of Namibia. Stockworks of fluorspar in granite are mined extensively at the Buffalo Mine in South Africa. Contact zones containing fluorspar are found in Morocco and South Africa. Greisen with fluorite, tin, tantalum, and columbium occurs in Jurassic granite in Nigeria. Pegmatites in Egypt, South Africa, Uganda, and Zaire contain fluorite but not in commercial quantities. Fluorite-bearing carbonatites associated with alkaline intrusive rocks are reported from Angola, Kenya, Malawi, Morocco, Mozambique, Namibia, South Africa, Tanzania, and Uganda. In South Africa, deposits of fluorspar are found disseminated in silicic granite in the Bushveld Complex and disseminated in alkaline intrusive rocks near Pretoria and Pilanesburg. Residual deposits derived from the weathering of stratiform replacement deposits in dolomite have yielded a small amount of fluorspar in the Marico district of South Africa. 
TABLE 1.-Fluorite in Africa

[Identified on pl. 1 by an italic number]

\begin{tabular}{|c|c|c|c|c|c|c|}
\hline $\begin{array}{l}\text { No. } \\
\text { on } \\
\text { pl. } 1\end{array}$ & $\begin{array}{l}\text { Mining district } \\
\text { or locality }\end{array}$ & $\begin{array}{l}\text { Latitude } \\
\text { (approxi- } \\
\text { mate }\end{array}$ & $\begin{array}{l}\text { Longitude } \\
\text { (approxi- } \\
\text { mate) }\end{array}$ & Geology & Remarks & References \\
\hline \multicolumn{7}{|c|}{ Algeria } \\
\hline $\mathrm{I}$ & Saharan Hoggar & $23^{\circ} 00^{\prime} \mathrm{N}$ & $6^{\circ} 30^{\circ} \mathrm{E}$ & $\begin{array}{l}\text { Fluorite vein with pitchblende in } \\
\text { Precambrian silicified granite. }\end{array}$ & - & DeKun, 1965. \\
\hline \multicolumn{7}{|c|}{ Angola } \\
\hline 1 & Coola & $12^{\circ} 31^{\prime} \mathrm{S}$ & $15^{\circ} 16^{\prime} \mathrm{E}$ & $\begin{array}{l}\text { Fluorite veins, lenticular bodies, and } \\
\text { replacements in carbonatite complex. }\end{array}$ & $\begin{array}{l}\text { Fluorite associated with barite, quartz, } \\
\text { and iron oxides. }\end{array}$ & $\begin{array}{l}\text { Lapido-Loureiro, } \\
\quad 1973 .\end{array}$ \\
\hline 2 & 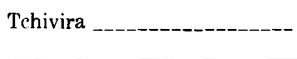 & $14^{\circ} 19^{\prime} \mathrm{S}$ & $13^{\circ} 53^{\prime} \mathrm{E}$ & $\begin{array}{l}\text { Fluorite in vugs and disseminated in } \\
\text { feldspar-carbonatite rock. }\end{array}$ & Discovered in 1966. & Do. \\
\hline \multicolumn{7}{|c|}{ Egypt } \\
\hline 1 & $\begin{array}{l}\text { Igla, Nabih, Nuweibeh, } \\
\text { Abu Dabbab, El Mueilha } \\
\text { tin-tungsten deposits. }\end{array}$ & $25^{\circ} 15^{\prime} \mathrm{N}$ & $34^{\circ} 35^{\prime} \mathrm{E}$. & $\begin{array}{l}\text { Fluorite in quartz veins and } \\
\text { pegmatites containing wolframite, } \\
\text { cassiterite, and topaz; in Precambrian } \\
\text { granitic and metamorphic rocks. }\end{array}$ & $\begin{array}{l}\text { Near east margin of Great Rift } \\
\text { system. }\end{array}$ & $\begin{array}{l}\text { Amin, } 1947 \text {; DeKun, } \\
1965 .\end{array}$ \\
\hline \multicolumn{7}{|c|}{ Kenya } \\
\hline 1 & $\begin{array}{l}\text { Kerio Valley area } \\
\text { (Elgeyo-Marakwet, } \\
\text { Baringo, and other } \\
\text { deposits near Eldoret; } \\
\text { also Kamnaon, Chof, and } \\
\text { Kimwarer deposits). }\end{array}$ & $\begin{array}{l}0^{\circ} 18^{\prime} \mathrm{N} .- \\
0^{\circ} 24^{\prime} \mathrm{N}\end{array}$ & $\begin{array}{l}35^{\circ} 35^{\prime} \mathrm{E} .- \\
35^{\circ} 38^{\prime} \mathrm{E}\end{array}$ & $\begin{array}{l}\text { Fluorspar veins in Miocene volcanic } \\
\text { rocks and Precambrian gneisses and } \\
\text { replacement deposits in Precambrian } \\
\text { crystalline limestone associated with } \\
\text { faults of the Rift Valley system; } \\
\text { epithermal. }\end{array}$ & $\begin{array}{l}\text { Reserves } 6 \text { million metric tons } \\
\text { proved, } 4 \text { million metric } \\
\text { tons probable, } 6 \text { million } \\
\text { metric tons inferred, all } 50 \\
\text { percent } \mathrm{CaF}_{2} \text {. Metallur- } \\
\text { gical.grade production by } \\
\text { gravity separation began in } \\
1972 \text {. Flotation plant } \\
\text { (100,000 t per year). Continental Ore } \\
\text { Conp. and Assoc. }\end{array}$ & $\begin{array}{l}\text { Mining Magazine, } \\
\text { 1972a; Nyambok } \\
\text { and Gaciri, } \\
\text { 1975; Reed and } \\
\text { Clarke, } 1976 .\end{array}$ \\
\hline 2 & Kakamaga-Margoli area & $0^{\circ} 10^{\prime} \mathrm{N}$ & $34^{\circ} 44^{\prime} \mathrm{E}$ & $\begin{array}{l}\text { Fluorite in gold veins near Precam- } \\
\text { brian granite. }\end{array}$ & - & DeKun, 1965. \\
\hline 3 & Rata area & $0^{\circ} 03^{\prime} \mathrm{S}$ & $34^{\circ} 32^{\prime} \mathrm{E}$ & $\begin{array}{l}\text { Fluorite in gold-bearing veins in } \\
\text { Precambrian rocks. }\end{array}$ & - & Do. \\
\hline 4 & $\begin{array}{l}\text { Homa-Ruri-Rangwa-Sokolo } \\
\text { area. }\end{array}$ & $0^{\circ} 31^{\prime} \mathrm{S}$ & $34^{\circ} 20^{\prime} \mathrm{E}$ & $\begin{array}{l}\text { Fluorite disseminated in carbonatites } \\
\text { in Miocene alkaline intrusive rocks. }\end{array}$ & - & $\begin{array}{l}\text { DeKun, 1965; Tuttle } \\
\text { and Gittins, 1966; } \\
\text { Le Bas, 1977. }\end{array}$ \\
\hline
\end{tabular}


TABLE 1.-Fluorite in Africa-Continued

\begin{tabular}{|c|c|c|c|c|c|c|}
\hline $\begin{array}{l}\text { No. } \\
\text { on } \\
\text { pl. } 1\end{array}$ & $\begin{array}{l}\text { Mining district } \\
\text { or locality }\end{array}$ & $\begin{array}{l}\text { Latitude } \\
\text { (approxi- } \\
\text { mate }\end{array}$ & $\begin{array}{c}\text { Longitude } \\
\text { (approxi- } \\
\text { mate) }\end{array}$ & Geology & Remarks & References \\
\hline \multicolumn{7}{|c|}{ Malawi } \\
\hline 1 & Chilwa Island _-_- & $15^{\circ} 19^{\prime} \mathrm{S}$ & $35^{\circ} 36^{\prime} \mathrm{E}$. & $\begin{array}{l}\text { Fluorite layers in carbonatite in } \\
\text { alkaline ring complex of Jurassic and } \\
\text { Cretaceous age near rift zone. }\end{array}$ & $\begin{array}{l}\text { Several layers } 2 \mathrm{~m} \text { thick, as } \\
\text { much as } 50 \mathrm{~m} \text { long, and } 25 \\
\text { percent fluorite. }\end{array}$ & Garson, 1965. \\
\hline 2 & Nkalonje & $15^{\circ} 34^{\prime} \mathrm{S}$ & $35^{\circ} 42^{\prime} \mathrm{E}$ & $\begin{array}{l}\text { Fluorite disseminated in carbonatite } \\
\text { complex (Jurassic and Cretaceous) } \\
\text { near rift zone. }\end{array}$ & $\begin{array}{l}\text { Quartz-fluorite layer } 0.3 \mathrm{~m} \\
\text { thick in brecciated fenite. } \\
\text { Explored in } 1972 \text { by Reynolds } \\
\text { International. }\end{array}$ & $\begin{array}{l}\text { Garson, 1965; Manos, } \\
\text { 1977. }\end{array}$ \\
\hline 3 & Songwe & $15^{\circ} 40^{\prime} \mathrm{S}$ & $35^{\circ} 50^{\prime} \mathrm{E}$ & -_-_do_-_-_-_-_-_- & $\begin{array}{l}\text { Apatite-fluorite rock in talus. } \\
\text { Boulders as much as } 0.6 \mathrm{~m} \\
\text { across, some } 75 \text { percent } \\
\text { fluorite. Explored by } \\
\text { Reynolds International in } \\
1973 .\end{array}$ & Do. \\
\hline 4 & Nsengwa & $15^{\circ} 24^{\prime} \mathrm{S}$ & $30^{\circ} 44^{\prime} \mathrm{E}$. & -_-_do & $\begin{array}{l}\text { Quartz-carbonatite, } 0.6 \mathrm{~m} \\
\text { thick, contains as much as } \\
50 \text { percent fluorite. }\end{array}$ & Garson, 1965 \\
\hline 5 & Kangkankunde & $15^{\circ} 07^{\prime} \mathrm{S}$ & $34^{\circ} 55^{\prime} \mathrm{E}$. & $\begin{array}{l}\text { Fluorite veins associated with carbon- } \\
\text { atite complex (Jurassic and } \\
\text { Cretaceous) near rift zone; locally } \\
\text { containing monazite, pyrochlore, } \\
\text { florencite, and bastnaesite. }\end{array}$ & $\begin{array}{l}\text { Quartz-fluorite bodies cut } \\
\text { Precambrian marble and ad- } \\
\text { jacent brecciated fenitized } \\
\text { gneiss over an area } 60 \times 9 \mathrm{~m} \text {; } \\
\text { <10 percent to } 50 \\
\text { percent fluorite. }\end{array}$ & $\begin{array}{l}\text { Garson, 1965; Smith, } \\
\quad 1956 .\end{array}$ \\
\hline \multicolumn{7}{|c|}{ Morocco } \\
\hline 1 & Ceuta area & $35^{\circ} 54^{\prime} \mathrm{N}$ & $5^{\circ} 22^{\prime} \mathrm{W}$ & $\begin{array}{l}\text { Fluorite gangue in lead-zinc veins } \\
\text { chiefly in Permian limestone. }\end{array}$ & 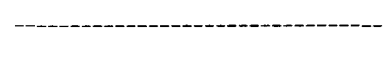 & $\begin{array}{l}\text { Morocco Service } \\
\text { Géologique, } 1969 .\end{array}$ \\
\hline 2 & Chaouene area & $35^{\circ} 08^{\prime} \mathrm{N}$ & $5^{\circ} 04^{\prime} \mathrm{W}$ & -_- do & -.-- & Do. \\
\hline 3 & Rio Bades-Casualidad area & $35^{\circ} 10^{\prime} \mathrm{N}$ & $4^{\circ} 07^{\prime} \mathrm{W}$ & _-_do & & Do. \\
\hline 4 & $\begin{array}{l}\text { Melilla area (Providencia } \\
\text { deposit). }\end{array}$ & $35^{\circ} 15^{\prime} \mathrm{N}$ & $2^{\circ} 57^{\prime} \mathrm{W}$ & $\begin{array}{l}\text { Fluorite with opal, gypsum, and } \\
\text { montmorillonite disseminated in } \\
\text { altered upper Tertiary rhyolite. }\end{array}$ & & DeKun, 1965. \\
\hline 5 & Ouezzane area & $34^{\circ} 43^{\prime} \mathrm{N}$. & $5^{\circ} 40^{\prime} \mathrm{W}$. & $\begin{array}{l}\text { Fluorite gangue in lead-zinc vein } \\
\text { between Triassic and Eocene beds. }\end{array}$ & & $\begin{array}{l}\text { Agard and Morin, } \\
1951 .\end{array}$ \\
\hline
\end{tabular}


6 Jebel Tirremi deposit near Taourirt).

$34^{\circ} 26^{\prime} \mathrm{N}$

$3^{\circ} 00^{\prime} \mathrm{W}$

$7 \quad$ Achèmeche area (El Hammam and othe deposits)

8 Jebel Zrahina deposit $32^{\circ} 52^{\prime} \mathrm{N} . \quad 5^{\circ} 56^{\prime} \mathrm{W}$

Fluorite veins and massive replacemen deposits containing quartz, calcite, barite, and local sulfides in Jurassic intrusive rocks.

Fluorite veins containing quartz and

calcite in Carboniferous limestone

belt $11 \mathrm{~km}$ long near upper Paleozoic grante; nearby fluorspar veins (Berganou, Gouaida, and Moufres st

vein in Paleozoic shale between Paleozoic granite.

9 Azegour molybdenum mine

$31^{\circ} 10^{\prime} \mathrm{N}$

$8^{\circ} 18^{\prime} \mathrm{W}$. Fluorite in molybdenum, copper, uranium contact-metamorphic deposit in Palsive reck trusive rock, accompanied by garnet, pyrite, scheelite, and pitchblende.

10 Tamazert

$32^{\circ} 30^{\prime} \mathrm{N} . \quad 4^{\circ} 54^{\prime} \mathrm{W} . \quad$ Fluorite disseminated in carbonatite complex also containing calcite, celestite, strontianite, witherite, barite, apatite, vermiculite,
pyrochlore, sulfides, and possibly pyrochlore
monazite.
Veins as much as $2 \mathrm{~m}$ thick contain $85-90$ percent $\mathrm{CaF}_{2}$ Reserves abou
metric tons.

Veins as much as $8 \mathrm{~m}$ thick. Proved reserves 3 millio metric tons 62 percent

CaF 2 ; probable reserves percent $\mathrm{CaF}_{2}$. Flotation

1958.

Agard and Morin,

1957. Permingeat

Carbonatites form dikes and

irregular masses cutting

Lissic limestones of the

Tuttle and Gittins, 1966

\section{Mozambique}

1 Chioco-Djanguire are

\section{$16^{\circ} 24^{\prime} \mathrm{S}$. \\ $32^{\circ} 35^{\prime} \mathbf{E}$. \\ Fluorite-quartz veins chiefly in Pre cambrian gneiss; some veins
basaltic and rhyolitic rocks.}

2 Changara (Mount Domba)

$16^{\circ} 46^{\prime} \mathrm{S} . \quad 33^{\circ} 02^{\prime}$ tic and rhyolitic rocks. area.

3 Canxixe (Mount Geramo) area southwest of

Fluorite-quartz veins along fault between Precambrian basement complex tween Precambrian basement conglomerate.

4 Macossa (Mount Djalira)

$17^{\circ} 38^{\prime} \mathrm{S} . \quad 34^{\circ} 13^{\prime} \mathrm{E}$.

luorite-quartz veins chiefly in Precambrian gneiss and granite; some in basalt and Karroo and post-Karroo

$17^{\circ} 56^{\prime} \mathrm{S} . \quad 34^{\circ} 12^{\prime}$ E. Fluorite-quartz veins in Precambrian gneiss and granulite; calcite and
chalcedony locally present.
Reserves containing more than

35 percent $\mathrm{CaF}_{2}-$ known,

possible 532,000 t. Bethlehem

possible $532,000 \mathrm{t}$.

Chermette, 1964;
U.S. Bureau of
Mines, 1973.

Reserves of $160,000 \mathrm{t}$ contain ing more than 35 percent

$\mathrm{CaF}_{2}$. Continental Ore

Reserves of $43,000 \mathrm{t}$ contain-

ing more than 35 percent

$\mathrm{CaF}_{2}$
Chermette, 1964.

Do.
Chermette 1964; U.S.

1973; Jolly, 1977 .

hermette, 1964; ines, 1973 . 


\begin{tabular}{ccccc}
\hline $\begin{array}{c}\text { No. } \\
\text { on } \\
\text { pl. 1 }\end{array}$ & $\begin{array}{c}\text { Mining district } \\
\text { or locality }\end{array}$ & $\begin{array}{c}\text { Latitude } \\
\text { (approxi- } \\
\text { mate }\end{array}$ & $\begin{array}{c}\text { Longitude } \\
\text { (approxi- } \\
\text { mate) }\end{array}$ & Geology \\
\hline
\end{tabular}

5 Mount Muambe

6 Xiluvo $16^{\circ} 34^{\prime} \mathrm{S} . \quad 34^{\circ} 12^{\prime} \mathrm{E}$ $19^{\circ} 15^{\prime} \mathrm{S} . \quad 34^{\circ} 04^{\prime} \mathrm{E}$.
Fluorite disseminated in Jurassic(?) carbonatite complex.

Fluorite in vugs in carbonatized Fluorite in vugs in carbonatized
volcanic breccia cut by carbonatite.
Mozamhique Serviços de Geologia Mi

Heinrich, 1966: Tuttle and Gittins

\section{Namibia (South West Africa)}

1 Okorusu-Marburg area, $20^{\circ} 06^{\prime} \mathrm{S} . \quad 16^{\circ} 46^{\prime} \mathrm{E}$ Otjiwarongo district.

Fluorite, quartz, apatite, hematite, and taniferous magnetite replacing Pre arbonatite in Precambrian graywack carbonatite in Precambrian graywack
faulted, brecciated, and domed up around margin of a nepheline plug. Also veins of fluorite and apatite. tin mine).

$20^{\circ} 20^{\prime} \mathrm{E} . \quad 13^{\circ} 50^{\prime} \mathrm{E}$

$21^{\circ} 15^{\prime} \mathrm{S} . \quad 15^{\circ} 35^{\prime} \mathrm{E}$

$3 \quad$ Kohero tin deposit and vicinity.

4 Omburo deposit $21^{\circ} 15^{\prime} \mathrm{S} . \quad 16^{\circ} 25^{\prime} \mathrm{E}$.

5 Kuibis (Aukam) deposit $26^{\circ} 48^{\circ}$ S. $\quad 16^{\circ} 50^{\prime} \mathrm{E}$

Fluorspar in irregular veins in Precambrian granite.

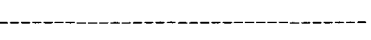

Fluorite, cassiterite, and sulfides in pipe in Precambrian limestone.

Fluorite in $0.3 \mathrm{~m}$ vein between tinbearing pegmatite and Precambrian granite. Fluorite disseminated in wolframite veins.
wo and quartz-tourma

Fluorite-quartz vein in marble lentils in a pendant in a Precambrian granite massif.

Production began in 1973 from flotation plant, $25,000 \mathrm{t}$ per year capacity. Closed
in 1975 by Gypsum Industries of South Africa.
U.S. Bureau of Mines, 1964: Hodge, 1973, Chermette, 1976 ; Verwoerd, 1967; Heinrich, 1966; Tuttle and Gittins, 966; Van Zijl, mall production of meta lurgical-grade fluorspar before 1964. Deposits conSteel Corp. and Tsumeb Corp. aciated with Bethlehem Steel Corp.

Sainsbury, 1964. Haughton and others
1939.

Chermette, 1964; 

6 Garub deposit, Warmbad $27^{\circ} 05^{\prime} \mathrm{S} . \quad 18^{\circ} 42^{\prime} \mathrm{E} . \quad$ Inclined breccia pipe of fluorspar in Precambrian granite gneiss, associated with galena, chalcopyrite, barite,
Production of about $1,900 \mathrm{t}$ of fluorspar.
Chermette, 1964;

\section{Nigeria}

1 Jos and Bukuru districts- $\quad 9^{\circ} 55^{\prime} \mathrm{N} . \quad 8^{\circ} 48^{\prime} \mathrm{E}$. Fluorite, cryolite, topaz, pyrochlore, cassiterite, columbite, tantalite, and greisens in Jurasic in stockwork and greisens in Jurassic
riebeckite soda-granites.

2 Akwan and Arufu lead-zinc

$7^{\circ} 50^{\prime} \mathrm{N} . \quad 9^{\circ} 12^{\prime} \mathrm{E} . \quad \begin{gathered}\text { riebeckite soda-granites. } \\ \text { Fluorite associated with galena }\end{gathered}$ sphalerite, calcite, siderite, barite and quartz in veins in Cretaceous limestone.

Somalia

Deposits in Benue trough, near rift zone.
Beer, 1952; Jacobson and others, 1958

Orajaka, 1973.

Farrington, 1952

Orajaka, 1973.

\begin{tabular}{|c|c|c|c|c|}
\hline & & & & Somalia \\
\hline \multirow[t]{2}{*}{1} & $\begin{array}{l}\text { Bihendulah Range } \\
\text { deposits. }\end{array}$ & $10^{\circ} 10^{\prime} \mathrm{N}$ & $45^{\circ} 08^{\prime} \mathrm{E}$. & $\begin{array}{l}\text { Fluorite associated with barite and } \\
\text { quartz in veins cutting Precambrian } \\
\text { gneiss. }\end{array}$ \\
\hline & & & & South Africa \\
\hline 1 & $\begin{array}{l}\text { Grobbelaars Hoek } \\
\text { deposit. }\end{array}$ & $23^{\circ} 18^{\prime} \mathrm{S}$ & $28^{\circ} 25^{\prime}$ E. & $\begin{array}{l}\text { Veins of fluorite cut by quartz in } \\
\text { Bushveld granite of Precambrian age }\end{array}$ \\
\hline 2 & $\begin{array}{l}\text { Leeuwbosch lead- } \\
\text { fluorspar deposit. }\end{array}$ & $24^{\circ} 28^{\prime} \mathrm{S}$ & $27^{\circ} 25^{\prime} \mathrm{E}$. & $\begin{array}{l}\text { Veins of fluorite and galena in } \\
\text { Precambrian dolomite. }\end{array}$ \\
\hline 3 & $\begin{array}{l}\text { Rietfontein-Ruigtepoort } \\
\text { area. }\end{array}$ & $24^{\circ} 45^{\circ} \mathrm{S}$ & $27^{\circ} 48^{\prime} \mathrm{E}$. & $\begin{array}{l}\text { Veins of fluorite, chlorite, } \\
\text { specularite, and quartz in post-Karro } \\
\text { normal faults; quartz-fluorite lenses } \\
\text { and pipes in quartz-chlorite greisen } \\
\text { in Bushveld granite. }\end{array}$ \\
\hline 4 & $\begin{array}{l}\text { Zwartkloof and other } \\
\text { deposits west of } \\
\text { Warmbad. }\end{array}$ & $24^{\circ} 36^{\prime} \mathrm{S}$ & $28^{\circ} 00^{\prime} \mathrm{E}$. & $\begin{array}{l}\text { Veins of fluorite and ankerite in Pre- } \\
\text { cambrian felsite; locally containing } \\
\text { chalcopyrite, pyrite, and sphalerite. }\end{array}$ \\
\hline
\end{tabular}

$-$

$-$

DeKun, 1965

(2)

DeKun, 1965

Do

Do.

Nearby tin deposits associated

with alkaline intrusive rocks

have fluorite as a gangue

mineral, especially near

Rooiberg. Small flotation

plant, 25,000 t per year

Deposits in northeast-trending

belt $40 \mathrm{~km}$ long. Resources

of 5 million metric tons 14

percent $\mathrm{CaF}_{2}$. Flotation
plant, capacity of $50,000 \mathrm{t}$

Production in 1971, first

full year of operation of

Zwartkloof mine was 43,000

cause ore grade was too low.
DeKun, 1965; Indus-

trial Minerals,

1970a, and South

African Mining

and Engineering

Journal, 1970

Crocker and

Martini, 1976. 
TABLE 1.-Fluorite in Africa-Continued

\begin{tabular}{lcccc}
\hline $\begin{array}{c}\text { No. } \\
\text { on }\end{array}$ & $\begin{array}{c}\text { Mining district } \\
\text { or locality }\end{array}$ & $\begin{array}{c}\text { Latitude } \\
\text { (approxi- } \\
\text { mate }\end{array}$ & $\begin{array}{c}\text { Longitude } \\
\text { (approxi- } \\
\text { mate) }\end{array}$ & Geology \\
South Africa_Continued
\end{tabular}

\section{South Africa-Continued}

5 Vischgat deposit

$24^{\circ} 33^{\prime}$ S. $28^{\circ} 36^{\prime}$ E. $\begin{gathered}\text { Veins of fluorite in Bushveld granite, } \\ \text { felsite, and pyroclastic rocks. }\end{gathered}$

6 Buffalo mine,

$24^{\circ} 28^{\prime} \mathrm{S} . \quad 28^{\circ} 40^{\prime} \mathrm{E}$. Fluorspar stockworks and veins as much as $0.6 \mathrm{~m}$ thick in leptite roof pendants in Bushveld granite; locally and bastnaesite.

7 Marico-Zeerust-Ottoshoop district (Wintershoek, tein, Buffelshoek, Gubbins mine, Oog van Malmanie)

$25^{\circ} 42^{\prime}$ S.- $\quad 26^{\circ} 02^{\prime}$ E.- $\quad$ Fluorspar mantos, pipes, veins, and irregular bodies replacing Precambrian dolomite; locally containing manganes chalcopyrite, pyrrhotite, tremolite, and talc.

8 Pilanesberg district Wydhoek and other deposits).

$25^{\circ} 14^{\prime} \mathrm{S}$. $\quad 27^{\circ} 05^{\prime} \mathrm{E}$. Fluorite associated with feldspar and apatite in veins in foyaite alkaline

$9 \quad$ Slipfontein deposit $25^{\circ} 05^{\prime} \mathrm{S} . \quad 27^{\circ} 15^{\prime} \mathrm{E} . \quad \begin{gathered}\text { Fluorite ring in quartz pipe in } \\ \text { Bushveld granite. }\end{gathered}$

10 Kromdraai and other deposits southwest of

$25^{\circ} 17^{\prime} \mathrm{S} . \quad 28^{\circ} 38^{\prime}$ E. Fluorite pipe containing hematite and manganese oxides in felsite, basalt, and agglomerate of Precambrian age;

Reserves of 60 million metric tons 16 percent $\mathrm{CaF}_{2}$ in and heavy-media plant with and heavy-media plant with filter cake per year.

Deposits in zone $60 \mathrm{~km}$ long. High-grade bodies worked
out: reserves $80-100$ millio metric tons 15 percent $\mathrm{CaF}_{2}$. Chemspar Limited (Phelps Dodge) and Marico Fluorspar Ltd. (U.S. Steel Corp.) have flotation plants with capacities of $100,000 \mathrm{t}$ respectively.

DeKun, 1965; Engineering and Mining

Holz, 1960; U.S. Bureau of Mines, zine $1972 \mathrm{~b}$; Holz 1972; Mining Magazine, 1973; Watson and Snyman, 1975; Crocker and
Martini, 1976.

DeVilliers, 1959; Engineering and 1972b, p. 194; Martini, 1976; Crocker and Martini 1976; Button, 1976 Von Gruenewaldt.

At Wydhoek, veins as much as $5 \mathrm{~m}$ thick contain fluorite apatite, and feldspar.

DeVilliers, 1959 DeKun, 1965.

DeVilliers, 1959

Kromdraai deposit covers area of $110 \times 64 \mathrm{~m}$; reserves of percent $\mathrm{CaF}_{2}$. Vergenoeg capacity of $95,000 \mathrm{t}$ of filter cake per year.

DeVilliers, 1959; Chermette, 1964;

DeKun, 1965; Crocker and
Martini, 1976. 
11 Pretoria district $25^{\circ} 33^{\prime} \mathrm{S}$.

$28^{\circ} 24^{\prime}$ E.

Fluorite, apatite, and pyrite in breccia

(Roodeplaat) and veins in alkaline

intrusive bodles (Walmansthal and

12 Hlabisa district,

$28^{\circ} 08^{\prime} \mathrm{S}$.

$31^{\circ} 54^{\prime} \mathrm{E}$.

Fluorite-quartz stockworks in early

Precambrian granite and post-
Carboniferous sandstone, shale,

and tillite.

13 Dyasonklip-Kenhardt

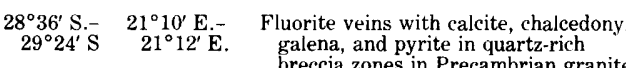
breccia zones in

14 Onseepkans-Styerkraal area.

$28^{\circ} 50^{\prime} \mathrm{S} . \quad 19^{\circ} 30^{\prime} \mathrm{E} . \quad$ Fluorite veins and pockets in pegmatites in Precambrian granite; locally
associated with scheelite.

15 Richtersveld deposits

$28^{\circ} 30^{\prime} \mathrm{S} . \quad 17^{\circ} 00^{\prime} \mathrm{E} . \quad$ Small deposits of fluorite in limestone near Precambrian granite and in gneiss.

16 Palabora (Loolekop) magnetite-rich carbonatite of the Palabora Complex, which cuts early
Precambrian granite and gneiss.

17 Glenover

$23^{\circ} 50^{\prime} \mathrm{S}$. $\quad 27^{\circ} 24^{\prime} \mathrm{E}$. Veins containing fluorite, barite, quartz, calcite, dolomite, pyrite, and specularite cut dolomitic carbonatite. Fluorite disseminated in apatite in phosphate breccia.

18 Goudini

$25^{\circ} 05^{\prime} \mathrm{S} . \quad 26^{\circ} 20^{\prime}$ E. Veinlets of fluorite, calcite, and rutile cut ankeritic carbonatite. Fluorite-dolomite veins.

19 Kruidfontein

$25^{\circ} 12^{\prime} \mathrm{S} . \quad 27^{\circ} 25^{\prime} \mathrm{E}$. Fluorite disseminated in carbonatite dikes and irregular bodies. Fluorite barite veins cut brecciated banded in volcanic rocks and carbonatite

$23^{\circ} 40^{\prime} \mathrm{S} . \quad 31^{\circ} 20^{\prime} \mathrm{E} . \quad$ Fluorite is a minor constituent of

DeVilliers, 1959

Deposits in belt $19 \times 20 \mathrm{~km}$; some veins several $\mathrm{km}$ long. Reserves 400,000

Kent and others, 1959; Chermette, 1964; Crocker and Martini, 1976.

Mineralized breccia zones a much as $3 \mathrm{~m}$ thick; high rachers.

DeVilliers, 1959 Hugo, 1963.

DeVilliers, 1959;

DeKun, 1965.

Kent and others, 1943; DeVilliers,

Carbonatite yields apatite, iron ore, vermiculite

Verwoerd, 1967; Heinrich, 1966; 1966 .

Veins average $0.3 \mathrm{~m}$ in thickness. Carbonatite is mined for phosphate.

Verwoerd, 1967; Tuttle and Gittins. 1966

Do.

\section{Southern Rhodesia}

1 Tinde deposit, Wankie

$18^{\circ} 19^{\prime} \mathrm{S}$.

Fluorite veins and fluorite-quartz-
chalcedony veins in Precambria granite.
Verwoerd, 1967; Heinrich, 1966 Tuttle and Gittins,

DeKun 1965; Mining Journal, 1968;
About $3 \mathrm{~km}$ of strike length of

veins known in 1968; $600 \mathrm{~m}$ explored, average width of

$5 \mathrm{~m}$. Proved reserves
$200,000 \mathrm{t}$. 
TABLE 1.-Fluorite in Africa-Continued

\begin{tabular}{|c|c|c|c|c|c|c|}
\hline $\begin{array}{l}\text { No. } \\
\text { on } \\
\text { pl. } 1\end{array}$ & $\begin{array}{l}\text { Mining district } \\
\text { or locality }\end{array}$ & $\begin{array}{l}\text { Latitude } \\
\text { (approxi- } \\
\text { mate }\end{array}$ & $\begin{array}{l}\text { Longitude } \\
\text { (approxi- } \\
\text { mate) }\end{array}$ & Geology & Remarks & Refierencess \\
\hline \multicolumn{7}{|c|}{ Sudan } \\
\hline 1 & Jebel Semeih deposit _-_-_-- & $12^{\circ} 44^{\prime} \mathrm{N}$ & $30^{\circ} 50^{\prime} \mathrm{E}$ & $\begin{array}{l}\text { Fluorite associated with quartz in } \\
\text { veins, joints, and irregular patches } \\
\text { in silicic granitic rock of Pre- } \\
\text { cambrian age. }\end{array}$ & $\begin{array}{l}\text { Discovered in } 1935 \text {. One } \\
\text { quartz-fluorite vein, } 123 \mathrm{~m} \\
\text { long and } 7 \mathrm{~m} \text { wide, contains } \\
11-42 \text { percent } \mathrm{CaF}_{2}, 56-86 \\
\text { percent } \mathrm{SiO}_{2} \text {, and less } \\
\text { than } 1 \text { percent } \mathrm{CaCO} \mathrm{S}_{3} \text {. Re- } \\
\text { serves } 100,000 \text { to } 300.000 \mathrm{t} \text {. }\end{array}$ & $\begin{array}{l}\text { Surdan Geological Sur- } \\
\text { vey, unpub. rep., } \\
1972 \text {; Whiteman, } \\
1971 \text {, p. 19-20. }\end{array}$ \\
\hline 2 & Jebel Dumbeir deposit _-_-_-_ & $12^{\circ} 35^{\prime} \mathrm{N}$ & $30^{\circ} 48^{\prime} \mathrm{E}$ & $\begin{array}{l}\text { Fluorite veins in fractured and faulted } \\
\text { Precambrian slate, quartzite, marble, } \\
\text { hornblende syenite, and red soda } \\
\text { granite; genetically associated with } \\
\text { the red soda granite. Fluorite also } \\
\text { replaces marble, impregnates quartzite, } \\
\text { and is disseminated in red soda } \\
\text { granite and hornblende syenite. }\end{array}$ & $\begin{array}{l}\text { Discovered in } 1971 \text {. Veins, } 25 \\
\text { cm to } 5 \mathrm{~m} \text { thick, contain as } \\
\text { much as } 95 \text { percent } \mathrm{CaF}_{2} \\
\text { and less than } 1 \text { percent each } \\
\text { of } \mathrm{SiO}_{2} \text { and } \mathrm{CaCO}_{3} \text {. } \\
\text { Fluorite disseminations and } \\
\text { impregnations contain less } \\
\text { than } 2 \text { yercent CaF } \\
\text { Reportedly associated with } \\
\text { carbonatite. }\end{array}$ & $\begin{array}{l}\text { Sudan Geological } \\
\text { Survey, unpul. } \\
\text { rep. 1972; } \\
\text { Whiteman, } 1971 . \\
\text { p. 19-20; Vail. } \\
1978, \text { p. 52. }\end{array}$ \\
\hline \multicolumn{7}{|c|}{ Swaziland } \\
\hline 1 & Hlatikulu deposite & $27^{\circ} 00^{\prime} \mathrm{S}$ & $31^{\circ} 18^{\prime} \mathrm{E}$ & $\begin{array}{l}\text { Fluorite-chalcedony-quartz-opal veins } \\
0.3-1.5 \mathrm{~m} \text { thick in Precambrian yranite } \\
\text { gneiss; fluorite in tin and molybdenum } \\
\text { deposits nearby. }\end{array}$ & Reserves of $1,600 \mathrm{t}$ & DeKun, 1965. \\
\hline \multicolumn{7}{|c|}{ Tanzania } \\
\hline & $\begin{array}{l}\text { Mbeya deposits (Panda } \\
\text { Hill and Sengeri Hill) }\end{array}$ & $8^{\circ} 59^{\prime} \mathrm{S}$. & $33^{\circ} 14^{\prime} \mathrm{E}$ & $\begin{array}{l}\text { Fluorite, pyrochlore, columbite, mag- } \\
\text { netite, cassiterite, barite, and } \\
\text { sulfide deposits in carbonatite } \\
\text { (Jurassic) in explosion vent and outer } \\
\text { ring-dike of extinct volcano (Panda); } \\
\text { accompanied by alkaline intrusive } \\
\text { rocks and next to rift valley. }\end{array}$ & $\begin{array}{l}\text { Fluorite veins cut carbonatite } \\
\text { dikes at. Sengeri Hill. }\end{array}$ & $\begin{array}{l}\text { Fawley and James, } \\
1955 ; \text { Heinrich, } \\
1966 .\end{array}$ \\
\hline 2 & Nyualla __- & $7^{\circ} 44^{\prime} \mathrm{S}$ & $32^{\circ} 42^{\prime} \mathrm{E}$ & $\begin{array}{l}\text { Fluorite in carbonatite dikelets and in } \\
\text { veins containing quartz, calcite, } \\
\text { barite, chalcopyrite, and galena. }\end{array}$ & - & $\begin{array}{l}\text { Heinrich, 1966: } \\
\text { Tuttle and Gittins, } \\
1966 .\end{array}$ \\
\hline \multicolumn{7}{|c|}{ Tunisia } \\
\hline 1 & $\begin{array}{l}\text { Djebel Sidi Abiod, } \\
\text { Cap Bon. }\end{array}$ & $37^{\circ} 02^{\prime} \mathrm{N}$ & $10^{\circ} 0 z^{\prime} \mathrm{E}$ & $\begin{array}{l}\text { Fluorite prospect in Tertiary sedimen- } \\
\text { tary rocks. }\end{array}$ & - & $\begin{array}{l}\text { U.S. Bureau of Mines, } \\
1953 \text {. }\end{array}$ \\
\hline
\end{tabular}

Djebel Sidi Abiod,
Cap Bon. $\quad 37^{\circ} 02^{\prime}$ N. $\quad 10^{\circ} 02^{\prime} \mathrm{E} . \quad \begin{gathered}\text { Fluorite prospect in Tertiary sedimen } \\ \text { tary rocks. }\end{gathered}$

S. Bureau of Mines 


\section{Zaghouan area (Hammam rriba, Djebilet el Kohol, Djebel Staa Djebel Ouest, and deposits).}

$36^{\circ} 24^{\prime}$ N.

$10^{\circ} 08^{\prime} \mathrm{E}$.

Fluorite veins and manto, or stratiform, replacement deposits in Jurassic and Liassic limestones near major fault zone; locally associated with barite Manto deposits contain 17-53 percent $\mathrm{CaF}_{2}$.

3 Bou Jaber deposit $35^{\circ} 45^{\prime} \mathrm{N}$ $8^{\circ} 23^{\prime}$

Fluorite and

in Cretaceous

\section{Uganda}

1 Singo deposits $0^{\circ} 40^{\prime} \mathrm{N} . \quad 32^{\circ} 00^{\prime} \mathrm{E} . \quad \begin{gathered}\text { Fluorite associated with tungsten in } \\ \text { Precambrian pegmatites. }\end{gathered}$

2 Mubende deposits

3 Buswale deposits

4 Bukusu

5 Tororo $0^{\circ} 34^{\prime}$ N. $\quad 31^{\circ} 24^{\prime}$ E. Fluorite in Precambrian granite

$0^{\circ} 23^{\prime} \mathrm{N} . \quad 33^{\circ} 54^{\prime} \mathrm{E}$. Fluorite in Precambrian granite

$0^{\circ} 50^{\prime} \mathrm{N} . \quad 34^{\circ} 18^{\prime} \mathrm{E}$. Fluorite disseminated in calcite

$0^{\circ} 42^{\prime} \mathrm{N} . \quad 34^{\circ} 12^{\prime} \mathrm{E} . \quad$ Fluorite disseminated in calcite
Hammam Zriba replacement depos-

it traced about $2 \mathrm{~km} ; 1-7 \mathrm{~m}$

thick; proved reserves of

6 million metric tons at $\mathbf{5 0}$

percent $\mathrm{CaF}_{2}$. For area,

sroved, probable, and pos-

metric tons. Two flotation

plants $(50,000$ and $5,000 \mathrm{t}$

per year). Industrie Chemique

de Fluor, Reynolds Inter-

national and Associates.

4 Kakontwe $10^{\circ} 59^{\prime}$ S. $26^{\circ} 40^{\prime}$ E. Fluorite in Precambrian limestone

\section{Zambia}

1 Kariba

$16^{\circ} 25^{\prime} \mathrm{S}$.
Fluorite, feldspar, and amethyst in
veins cutting Precambrian gneiss on faults.

U.S. Bureau of Mines, 1953; Sainfeld, 1956; Chermette, 1958; U.S. Burea of Mines, 1972:

Thibi, 1973

Merwin, 1976.

Sainfeld, 1956.

DeKun, 1965

Do.

Do.

Heinrich, 1966.

Heinrich, 1966;

DeKun, 1965

Do.

Do.

Do.

Hitchon, 1957 
The deposits range in size from mere occurrences of fluorite to the deposits of the Marico district, South Africa, which contain more than 80 million metric tons of fluorspar ore (about 13.5 million metric tons of $\mathrm{CaF}_{2}$ ) and may be the largest fluorspar deposit in the world. Table 1 gives reserve figures for many of the deposits, and plate 1 shows by size of the symbols whether a fluorspar deposit is large ( $>1$ million metric tons), medium $(1,000,000-50,000$ t), or small $(<50,000$ t). A medium-size deposit in Africa might have about $100,000 \mathrm{t}$ of contained $\mathrm{CaF}_{2}$, which is slightly less than 10 percent of the tonnage consumed annually in the United States. Plate 1 shows few medium-size deposits, which are all veins or stockworks and pipes; their scarcity probably results from the lack of exploration and development work at many of the small deposits shown or from the lack of published information.

The nine large deposits are in South Africa (Marico, Buffalo, Kromdraai, Zwartkloof), Namibia (Okorusu), Kenya (Kerio Valley), Morocco (El Hammam, Jebel Tirremi), and Tunisia (Zaghouan). These major deposits occur as mantos or replacements, veins, or stockworks and pipes.

\section{FLUORSPAR PRODUCTION AND EXPORTS}

Fluorspar mining on a large scale in Africa is a relatively young and

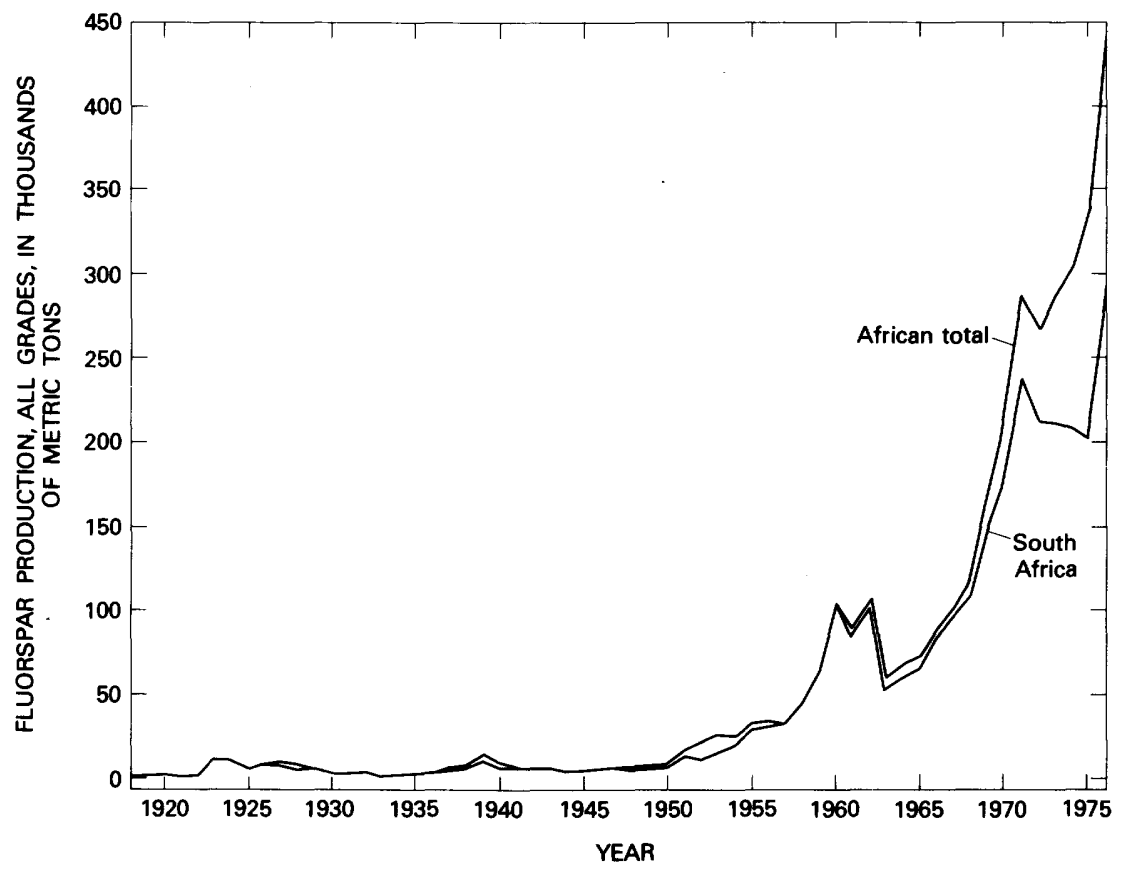

FIGURE 1.-Comparison of fluorspar production from South Africa with total African fluorspar production, 1918-76. 


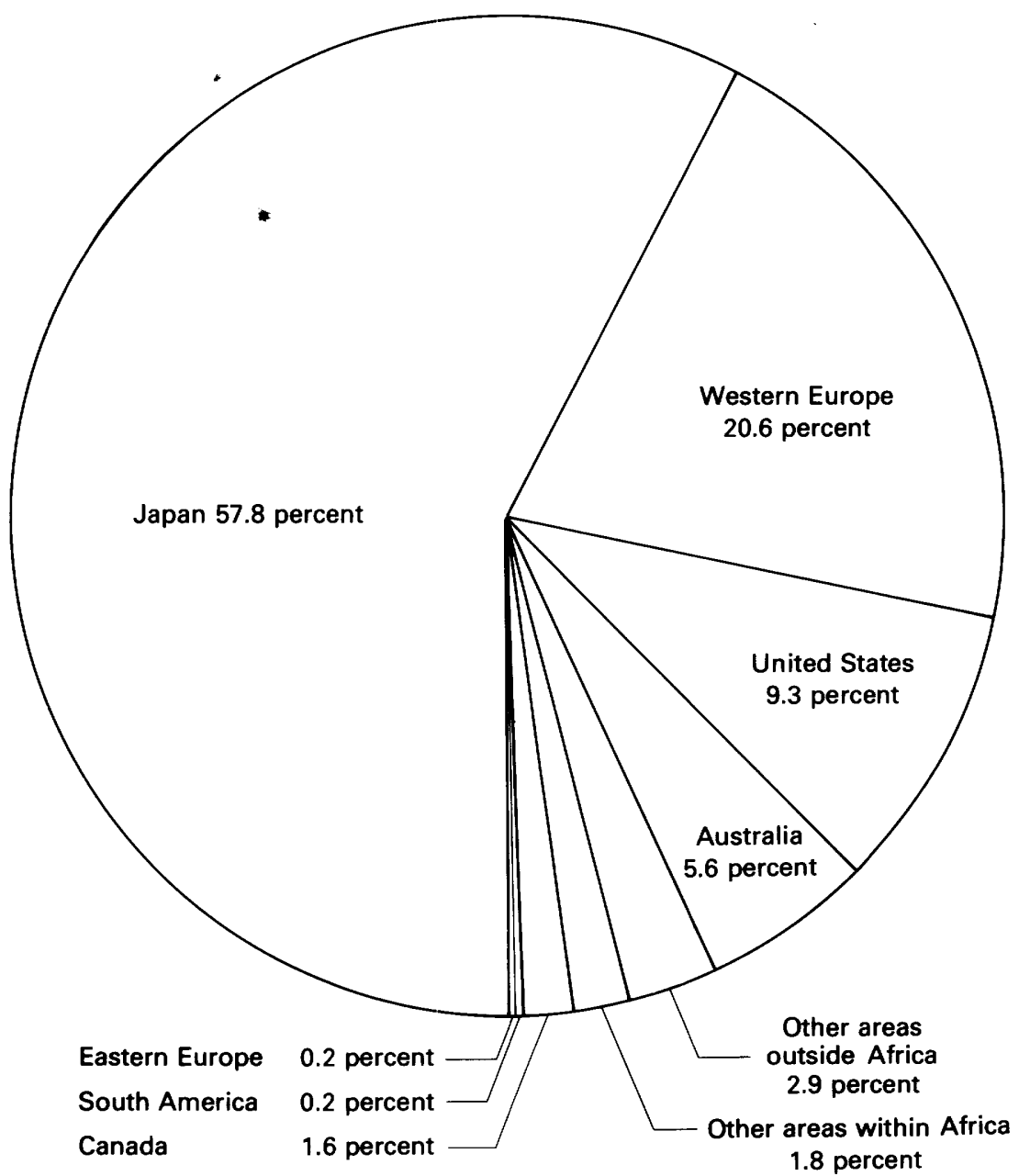

FIgURE 2. - African fluorspar exports 1958-73 by area of destination.

growing industry (see table 2); production is expected to increase. African production began in 1918 in the Zeerust area of South Africa but did not exceed an annual rate of 100,000 t until 1960 (fig. 1). Fluorspar production from Africa through 1976 totals about 3.5 million metric tons. Approximately 88 percent of this total was produced since 1960 , and 80 percent originated in South Africa. About 83 percent of African fluorspar production in 1976 was exported. Figure 2 shows the destination of African fluorspar exports between 1958 and 1973. Fluorspar production in Africa in 1976 amounted to 453,115 t, about 10 percent of the world output. South African fluorspar production in 1976 was 290,718 t or about 6 percent of the world production, as com- 
TABLE 2. - Selected data on African fluorspar production through 1976

[Most of the data are from the U.S. Bureau of Mines, Mineral Resources of the United States, 1925-31, and Minerals Yearbooks, 1932-75. Short tons converted to metric tons]

\begin{tabular}{|c|c|c|c|c|c|}
\hline Country & $\begin{array}{c}\text { Productive } \\
\text { years }\end{array}$ & $\begin{array}{c}\text { Total } \\
\text { production } \\
\text { (metric tons) }\end{array}$ & $\begin{array}{c}\text { Year of } \\
\text { maximum } \\
\text { production }\end{array}$ & $\begin{array}{c}\text { Maximum annual } \\
\text { production } \\
\text { (metric tons) }\end{array}$ & $\begin{array}{l}\text { Percent } \\
\text { exported } \\
\text { in } 1974\end{array}$ \\
\hline & & & & . & \\
\hline Egypt & $1970-76$ & 6,797 & 1973 & 1,509 & 0.0 \\
\hline Kenya & $1968-76$ & 239,116 & 1976 & 75,027 & 96.2 \\
\hline Morocco & ${ }^{1} 1948-76$ & 148,018 & 1976 & 51,450 & 28.9 \\
\hline Namibia _..... & ${ }^{1} 1927-63$ & 18,317 & 1952 & 4,418 & .0 \\
\hline South Africa & $1918-76$ & $2,836,137$ & 1976 & 290,718 & 59.5 \\
\hline Southern Rhodesia & ${ }^{1} 1938-76$ & 5,583 & 1956 & 855 & .0 \\
\hline Tunisia & ${ }^{1} 1937-76$ & 291,609 & 1973 & 46,600 & 90.3 \\
\hline Zambia __- & $1973-76$ & 969 & 1975 & 499 & .0 \\
\hline Total __- & $-----\infty$ & $3,546,546$ & $-\ldots$ & ------ & -- \\
\hline
\end{tabular}

${ }^{1}$ Intermittent.
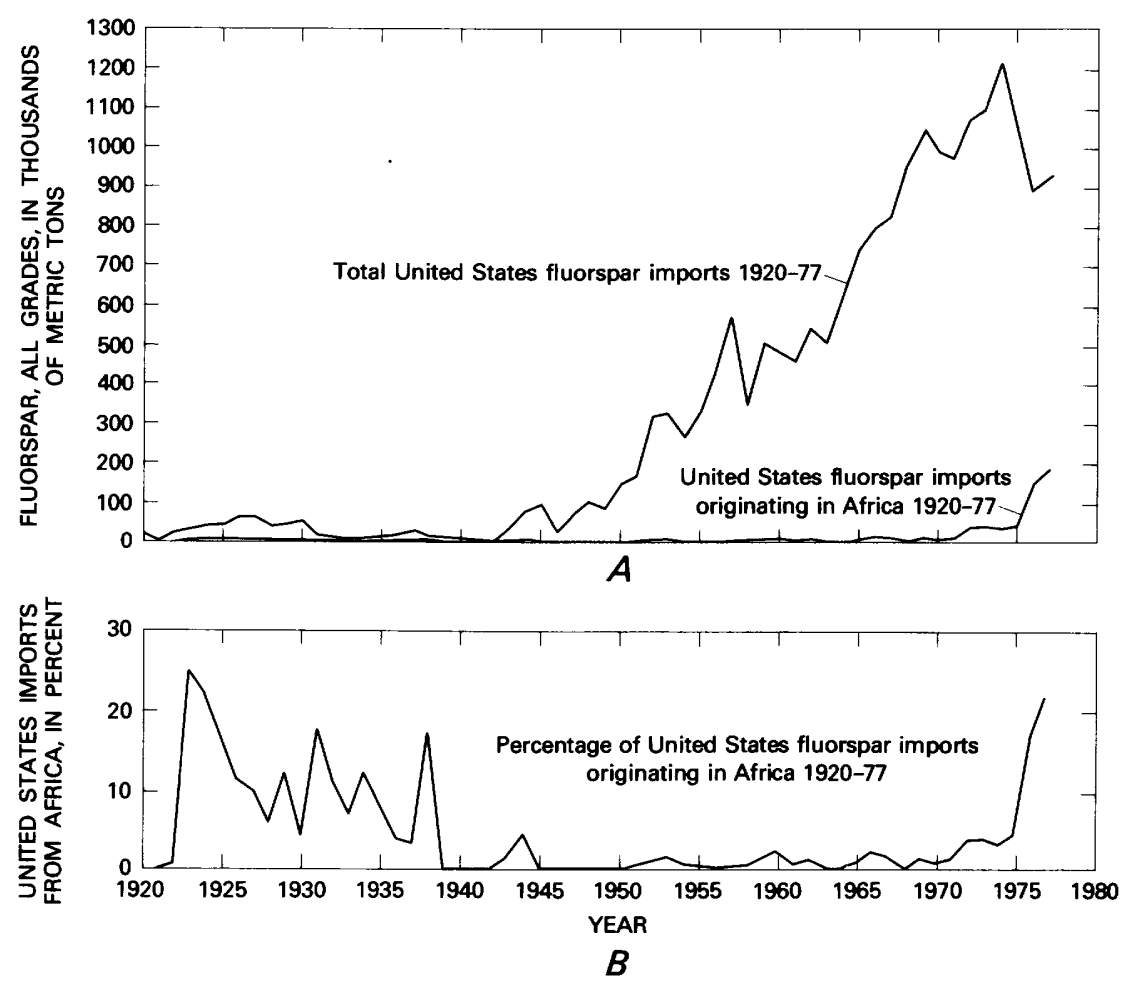

Figure 3.-Amount of fluorspar imported by the United States. $A$, Total imports of fluorspar; imports of fluorspar from Africa. $B$, The percentage of imports of fluorspar from Africa, 1920-77. 
pared with United States production of about $169,000 \mathrm{t}$ in that year.

Figure 3 shows the total U.S. imports of fluorspar, U.S. imports of fluorspar from Africa, and the percentage of U.S. imports of fluorspar from Africa for the years 1920 through 1977. The sharp increase in our fluorspar imports from Africa since 1975 is impressive and reflects our greater reliance upon South Africa as a source.

\section{FLUORSPAR RESERVES}

Estimates of African fluorspar reserves of proved and probable ore total about 192 million metric tons, averaging 22 percent $\mathrm{CaF}_{2}$. South Africa accounts for about 86 percent of this total and ranks first in world reserves of fluorspar. These estimates are taken from reports published in 1976 or later and are shown by country and district in table 1. Table 3 shows the estimates of combined proved and probable, and also possible, fluorspar ore reserves by country.

The total reserves, when calculated as 35 percent $\mathrm{CaF}_{2}$ or equivalent, are about 40 percent of the fluorspar reserves of the world, although most world reserve figures do not include estimates for fluorspar deposits averaging much lower than 30 percent $\mathrm{CaF}_{2}$. The large size of some South African deposits and the low production costs permit largescale mining of reserves that are of a lower grade than can be mined elsewhere. According to a Stanford Research Institute estimate, the cost in South Africa to produce one ton of acid-grade concentrate from a low-grade fluorspar ore is $\$ 55$ compared with a cost of $\$ 94$ in the United States (Quan, 1978, p. 20-21).

The African deposits are not described in the literature in sufficient detail to evaluate these reserve estimates or to make estimates of other categories of fluorspar resources.

TABLE 3.-African fluorspar reserves, in millions of metric tons

\begin{tabular}{|c|c|c|c|c|}
\hline Country & $\begin{array}{l}\text { Proved and } \\
\text { probable } \\
\text { ore }\end{array}$ & $\begin{array}{l}\text { Percentage } \\
\text { of total }\end{array}$ & $\begin{array}{c}\text { Possible } \\
\text { ore }\end{array}$ & $\begin{array}{c}\text { Approximate } \\
\text { grade } \\
\text { (percent } \mathrm{CaF}_{2} \text { ) }\end{array}$ \\
\hline Kenya _-_-_-_-_-_- & 10 & 5.2 & 6 & 50 \\
\hline Morocco & 6 & 3.1 & 2 & 50 \\
\hline Mozambique & .4 & .2 & .5 & 35 \\
\hline Namibia & 6 & 3.1 & 2 & 50 \\
\hline South Africa & ${ }^{1} 160.4$ & 83.3 & $4 \overline{1}$ & 17 \\
\hline Tunisia & 9 & 4.7 & 3 & 50 \\
\hline Others ${ }^{2}$ & .7 & .4 & .3 & 35 \\
\hline Totals & 192.5 & 100.0 & 54.8 & -- \\
\hline Average grade & - & $\ldots$ & $\ldots$ & 22 \\
\hline
\end{tabular}

1 Compares with 157 million metric tons of measured ore reserves containing 20 percent $\mathrm{CaF}_{2}$ estimated by Gössling (1978).

${ }^{2}$ Chiefly Southern Rhodesia and Sudan. 


\section{OUTLOOK FOR FUTURE SUPPLY}

Africa is able to provide an increasingly significant share of the world's fluorspar supply. The extensive reserves of proved and probable ore are equivalent to about a 75-year supply at the 1976 rate of production, although the largest deposit, the Marico district, South Africa, probably will not be depleted during that period. Geological conditions are favorable for the discovery of additional major deposits.

Until steel, aluminum, and fluorine chemical industries are developed in more African countries, most of the African fluorspar will continue to be available for export. South Africa, the only substantial consumer of fluorspar in Africa, in 1976 used only about 39,000 t.

An annual growth rate for African fluorspar production cannot be reliably predicted, even for the next few years, mainly because of worldwide recession, decreasing production of fluorocarbons for aerosol use, decrease in use of fluorides in the aluminum industry, and the growing use of fluorine from phosphate rock. The capacity of existing fluorspar plants in Africa is about 755,000 t of fluorspar per year (table 1). This capacity would permit a possible increase in production of about 60 percent above the 1976 rate. Gössling (in Industrial Minerals, 1979) predicted that the South African fluorspar industry will continue expansion over the next 7 years to an annual production level of $560,000 \mathrm{t}$, that 85-90 percent will be exported, and that South Africa will become the world's largest fluorspar producer.

Increased production and exports of lower-cost fluorspar from Africa probably will have adverse effects upon the world fluorspar industry. Some smaller higher-cost mining operations may be closed, unless demand for fluorspar grows at a faster rate. World demand for fluorspar should increase as economic conditions improve and as developing countries require a fluorspar supply for any new steel, aluminum, or fluorine chemical industries.

\section{SEARCH FOR ADDITIONAL DEPOSITS}

The most promising places to search for new deposits in Africa are near the major deposits and in the areas having the most numerous deposits (pl.1). Areas near carbonatites, alkaline and highly silicic igneous rocks, and adjacent rocks, especially where there may be readily replaceable carbonate rocks, are the most favorable sites for large deposits containing more than 1 million tons of fluorspar. The areas of carbonatites and other alkaline extrusive and intrusive bodies commonly are in regions of tension marked by abyssal or subcrustal fractures, rifts, block faults, grabens, and mainly vertical movement (Mitchell and Garson, 1976).

Carbonatites are more abundant in Africa than in any other continent. Africa has about three times more deposits than does Asia, the continent in second-place (Heinrich, 1966). Fluorite has been reported 
in 24 of the 115 carbonatite localities of Africa shown on plate 1. Probably careful search of the other 91 carbonatite localities would increase the number of known localities that are associated with fluorite, possibly some containing commercial deposits. Most of the list of carbonatite localities (table 4) was obtained from publications by Pecora (1956), Smith (1956), Garson (1965), Heinrich (1966), Tuttle and Gittins (1966), Verwoerd (1967), Lapido-Loureiro (1973), Blanchot (1975), and Le Bas (1977).

The only extensive fluorspar deposit of commercial grade associated with carbonatites in Africa is the Okorusu deposit in Namibia (Van Zijl, 1962), but other carbonatites also contain fluorite and are considered to be possible sources of commercial fluorspar. Carbonatites of Africa may also contain valuable deposits of pyrochlore (a fluoroniobite of calcium and sodium), phosphate, vermiculite, calcite, barite, nepheline, strontianite, thorium, uranium, rare earths, titanium, magnetite, copper, and zirconium. The fluorite may occur in vugs, layers, and disseminated in the carbonatite or adjacent rocks; in late-stage replacement deposits in carbonatite, alkaline intrusive rock, or country rock; or in veins containing quartz, calcite, barite, apatite, and sulfide minerals. Fluorine minerals, other than fluorite, found in carbonatites include pyrochlore, fluorapatite, fluorphlogopite, bastnaesite, and sellaite $\left(\mathrm{MgF}_{2}\right)$.

Carbonatites occur in alkaline petrologic provinces and in Africa range in age from Precambrian to Holocene. They are generally small bodies compared with other masses of igneous rock, but because they commonly are circular or elliptical domed features rising above the surrounding terrain, most should be detectable on aerial photographs or satellite imagery. Probably many more carbonatites and fluorspar deposits will be found in Africa, for much of the continent has not been geologically mapped in detail, and in large areas, the bedrock is concealed by wind-blown sand and other surficial deposits.

Le Bas (1977) recognized the following four-stage sequence of intrusions in the formation of carbonatites: (1) magmatic sövitic carbonatite consisting of coarse-grained calcite; (2) minor intrusions of alvikitic carbonatite consisting of fine-grained ferroan calcite, apatite, magnetite, limonite, and goethite; (3) ferruginous dark-colored carbonite containing fluorite; and (4) low-temperature veins of calcite carbonatite containing fluorite, barite, and quartz. Fluorite may have formed in any of the four stages, but deposits of commercial quantity and grade most likely formed during the last two stages.

Alkaline petrologic provinces of Africa, in which carbonatites or fluorite deposits have not yet been reported to be associated with the alkaline rocks, are found in Chad, Ethiopia, Malagasy Republic, Mali, Niger, and Somalia (Beer, 1952). Alkaline igneous rocks were reported 
TABLE 4.-Carbonatite localities of Africa

[Italics indicate that fluorite has been reported with the carbonatites]

\section{ANGOLA}

1. Cuacra

2. Catanda

3. Capuia

4. Bailundo

5. Monte Verde

6. Coola

7. Chianga

8. Longonjo

9. Capunda

10. Bonga

11. Tchivira

12. Virulundo

13. Morro Vermelho

14. Lupongola (Chitado)

\section{ETHIOPIA}

1. Bishoftu

\section{KENYA}

1. Tinderet

2. Buru

3. Homa

4. Rangwa

5. Ruri

6. Sokolo

7. Mrima

\section{MALAWI}

1. Lake Malombe vents

2. Kadongosi

3. Chaumbwi

4. Nailuwa

5. Kangankunde

6. Kalambo Stream

7. Mongolowe

8. Palula

9. Kapiri

10. Mtsimukwe

11. Chilwa Island

12. Nsengwa

13. Tundulu

14. Nkalonje

15. Songwe

16. Bangala

17. Namangali

\section{MALI}

1. Adrar Tadhak

\section{MAURITANIA}

1. Richat

2. Tabrinkout

3. Akjoujt

4. Legleitat

\section{MOROCCO}

1. Tamazert

\section{MOZAMBIQUE}

1. Lipuche

2. Cone Negose

3. Muambe

4. Chuara

5. Chandava

6. Xiluvo

7. Cura

\section{NAMIBIA}

1. Swartbooisdrif

2. Epembe

3. Okorusu

4. Ondurakorume

5. Kalkfeld

6. Osongombo

7. Brukkaros

8. Chamais

\section{SOMALIA}

1. Darkainle

\section{SOUTH AFRICA}

1. Palabora (Loolekop)

2. Glenover

3. Magnet Heights

4. Spitskop

5. Nooitgedacht

6. Goudini

7. Kruidfontein

8. Bulhoek

9. Tweeriver

10. Zoutpan

11. Roodeplaat

12. Derdepoort

13. Salpeterkop

\section{SOUTHERN RHODESIA}

1. Katete Hill

2. Dorowa

3. Shawa

4. Chishanya

\section{TANZANIA}

1. Mosonik

2. Oldoinyo Lengai

3. Kerimasi

4. Sadiman

5. Meru

6. Burko

7. Monduli-Arusha

8. Oldoinyo Dili 
TABLE 4.-Carbonatite localities of Africa-Continued

TANZANIA-Continued

9. Essimingor

10. Galappo

11. Basotu

12. Hanang and Balangida

13. Sangu

14. Wigu Hill

15. Maji Ya Weta

16. Ngualla

17. Luhombero

18. Songwe Scarp

19. Musensi

20. Mbeya (Panda and Sengeri)

21. Nachendazwaya

\section{UGANDA}

1. Toror Hills

2. Napak
3. Lolekek

4. Budeda

5. Bukusu

6. Fort Portal

7. Tororo

8. Sukulu

\section{ZAIRE}

1. Bingu

2. Lueshe

3. Kawezi

\section{ZAMBIA}

1. Nkombwa

2. Kaluwe

3. Nachomba

4. Mwambuto

5. Chasweta

in about 80 percent of the 19 African countries having fluorite deposits (table 1). The alkaline rocks include nepheline syenites, foyaites, syenites, ijolites, fenites, and riebeckite granites. The riebeckite granites and other alkaline granites of Nigeria contain a variety of fluorine minerals, including accessory fluorite, cryolite, thomsenolite, topaz, pyrochlore, and riebeckite; there has been no production of fluorspar there, but cassiterite and columbite have been recovered from the decomposed granite (Williams and others, 1956).

Saline lakes and playas of the rift valleys of Africa may contain fluorine minerals of commercial importance. Fluorine hot springs near the rifts, hydrothermal solutions, and also the eroded carbonatites and associated alkaline and silicic intrusive and extrusive rocks rich in fluorine may have contributed fluorine to the basins. Saline water from Lakes Hannington, Magadi, Nakuru, and Elmenteita, all in the rift valley of southwestern Kenya, contain $1,170,1,270,1,400$, and 1,627 parts per million of fluorine, respectively (Baker, 1958, p. 58; Hay, 1966, p. 33). Lake Magadi has large commercial deposits of trona that locally contains as much as 22 percent villiaumite $(\mathrm{NaF})$ and 6 percent fluorite (Sheppard and Gude, 1969, p. D73). Possibly fluorite will be found in an environment of Cenozoic tuffaceous lake beds near the rift zones; deposits of this type occur in Pleistocene sediments near Rome, Italy (Spada, 1969), some beds averaging 55 percent $\mathrm{CaF}_{2}$, and in Pliocene beds of lower grade near Rome, Ore., (Sheppard and Gude, 1969) and Eastgate, Nev. (Sheppard and Gude, 1976).

\section{REFERENCES CITED}

Agard, Jules, and Morin, Ph., 1951, Les gisements de fluorine du Maroc: Morocco Service Géologique Notes et Mémoires no. 83, p. 184-219. 
Amin, M. S., 1947, A tin-tungsten deposit in Egypt: Economic Geology, v. 42, no. 7, p. 637-671.

Baker, B. H., 1958, Geology of the Magadi area; degree sheet 51, S.W. quarter: Kenya Geological Survey Report 42, $81 \mathrm{p}$.

Beer, K. E., 1952, The petrography of some of the riebeckite granites of Nigeria: Great Britain Geological Survey and Museum, Atomic Energy Division Report 116, 38 p.

Blanchot, A. J., ed., 1975, Plan minéral de la République Islamique de Mauritanie: [France] Bureau de Recherches Géologiques et Minières, $554 \mathrm{p}$.

Button, Andrew, 1976, Transvaal and Hamersley Basins-review of basin development and mineral deposits: [South Africa] Minerals Science and Engineering, v. 8, no. 4, p. 262-293.

Chermette, Alexis, 1958, Regards sur la production mondiale de spath fluor-perspectives d'avenir: extracted from l'Echo des Mines et Métallurgie, nos. de Juin 1957 à Juin 1958, 33 p. (Some parts differ from original.)

-1964, Le spath-fluor en Afrique du Sud: no. 3583, p. 187-192; no. 3584, p. 243-245; no. 3585, p. 301-303; no. 3586, p. 349-352; and no. 3587, p. 401-402. 1976, L'evolution de la production mondiale de spath-fluor, part 2: Mines et Métallurgie, no. 126, p. 51-55.

Choubert, Georges, and Faure-Muret, Anne, 1972-1975, Geological World Atlas, Maps 6, 7, 8: Paris UNESCO, scale 1:10,000,000.

Crocker, I. T., and Martini, J. E. J., 1976, Fluorspar, in Mineral resources of the Republic of South Africa (5th ed.): South Africa Geological Survey Handbook 7, p. 357-363.

DeKun, Nicolas, 1965, The mineral resources of Africa: Amsterdam, Netherlands, Elsevier Publishing Co., 740 p.

De Villiers, John, 1959, compiler, Fluorspar, in The mineral resources of the Union of South Africa (4th ed.): South Africa Geological Survey Handbook 1, p. 404-414.

Engineering and Mining Journal, 1972a, Geologic and mineral maps of South Africa: v. 173 , no. 11 , p. $105-116$.

-1972b, Asbestos and fluorspar - both have growth potential: v. 173, no. 11, p. 194. 1978, Fluorspar market: v. 179, no. 1, p. 56.

Farrington, J. L., 1952, A preliminary description of the Nigerian lead-zinc field: Economic Geology, v. 47, no. 6, p. 583-608.

Fawley, A. P., and James, T. C., 1955, A pyrochlore (columbium) carbonatite, southern Tanganyika: Economic Geology, v. 50, no. 6, p. 571-585.

Garson, M. S., 1965, Carbonatites in southern Malawi: Malawi Geological Survey Bulletin 15, p. 108-110.

Gössling, H. H., 1978, Fluorspar: 1973 to 1980, a commodity profile: South Africa Department of Mines, Minerals Bureau Report 3, 36 p.

Haughton, S. H., Frommurze, H. F., Gevers, T. W., Schwellnus, C. M., and Rossouw, P. J., 1939, The geology and mineral deposits of the Omaruru area, South West Africa; an explanation of sheet no. 71: South Africa (Union of) Department of Mines, Geological Survey, $151 \mathrm{p}$.

Hay, R. L., 1966, Zeolites and zeolitic reactions in sedimentary rocks: Geological Society of America Special Papers 85, $130 \mathrm{p}$.

Heinrich, E. W., 1966, The geology of carbonatites: Chicago, Rand McNally, 555 p.

Hitchon, Brian, 1957, The geology of the Kariba area: Northern Rhodesia (Zambia) Geological Survey Report 3, $41 \mathrm{p}$.

Hodge, B. L., 1973, World fluorspar developments-2: Industrial Minerals, no. 69, p. 9-21.

Holz, Peter, 1960, Fluorspar in South Africa: Pit and Quarry, v. 52, no. 11, p. 172-175. 1972, New fluorspar plant completed at Naboomspruit: South African Mining and Engineering Journal, v. 84, no. 4075, p. 11-13.

Hugo, P. J., 1963, Fluorspar deposits on Pyp Klip West and Wit Vlei, Kenhardt district, Cape Province: South Africa Geological Survey Annals, v. 1 (1962), p. 119-126. 
Industrial Minerals, 1970a, Zwartkloof fluorspar plant on schedule: no. 29, p. 45.

-1970 b, Morocco-further fluorspar potential: no. 38, p. 33.

1971, Morocco-U.K. and Preussag finalise fluorspar agreement: no. 49, p. 30.

1978, South Africa, Fluorspar optimism: no. 133, p. 15-16.

Jacobson, R. R. E., MacLeod, W. N., and Black, Russell, 1958, Ring-complexes in the younger granite province of northern Nigeria: Geological Society of London Memoir $1,72 \mathrm{p}$.

Jolly, J. H., 1976, The mineral industry of the Republic of South Africa: U.S. Bureau of Mines Minerals Yearbook 1973, v. 3, p. 745-769.

Jolly, J. L. W., 1977, The mineral industry of Angola, Mozambique, and Guinea Bissau: U.S. Bureau of Mines Minerals Yearbook 1974, v. 3, p. 85-98.

Jones, D. L., Robertson, I. D. M., and McFadden, P. L., 1975, A paleomagnetic study of Precambrian dyke swarms associated with the Great Dyke of Rhodesia: Geological Society of South Africa Transactions and Proceedings, v. 78, pt. 1, p. 57-65.

Kent, L. E., Russell, H. D., and Van Rooyen, D. P., 1943, Fluorspar in the Union of South Africa and South West Africa: South Africa (Union of) Department of Mines, Geological Series Bulletin 14, $69 \mathrm{p}$.

Lapido-Loureiro, F. Eduardo, 1973, Carbonatitos de Angola: Luanda, Instituto de Investigação Cientifica de Angola, v. 11, 242 p.

Le Bas, M. J., 1977, Carbonatite-nephelinite volcanism, an African case history: New York, John Wiley, $347 \mathrm{p}$.

Manos, Anthony, 1977, Fluorspar, in Industrial Minerals of Malawi: Industrial Minerals, no. 123 , p. $62-63$.

1978, Industrial minerals of Rhodesia: Industrial Minerals, no. 126, p. 93-107.

Martini, J. E. J., 1976, The fluorite deposits in the Dolomite Series of the Marico district, Transvaal, South Africa: Economic Geology, v. 71, no. 3, p. 625-635.

Merwin, R. W., 1976, The mineral industry of Tunisia: U.S. Bureau of Mines Minerals Yearbook 1973, v. 3, p. 847-854.

Mining Journal, 1968, Rhodesia, in Mining Annual Review: May 1968, p. 293.

Mining Magazine, 1972a, Kenyan fluorspar source: v. 127, no. 5, (Nov.), p. 439.

-1972b, Buffalo expansion: v. 127, no. 5 (Nov.), p. 483.

-1973, South Africa's Buffalo fluorspar in full production: v. 129, no. 6 (Dec.), p. 501 .

Mitchell, A. H. G., and Garson, M. S., 1976, Mineralization at plate boundaries: [South Africa] Minerals Science and Engineering, v. 8, no. 2, p. 129-170.

Morocco Service Géologique, 1969, Carte des mineralisations plombo-zinciferes du Maroc: Morocco Service Géologique, Notes et Mémoires no. 215, scale 1:200,000.

Mozambique Serviços de Geologia e Minas, 1974, Carta de jazigos é ocorrências Minerais: Lorenzo Marques, scale 1:2,000,000 and Mozambique Serviços de Geologia e Minas, Noticia explicativa, p. 35.

Nyambok, I. O., and Gaciri, S. J., 1975, Geology of the fluorite deposits in Kerio Valley, Kenya: Economic Geology, v. 70, no. 2, p. 299-307.

Orajaka, S. O., 1973, Possible metallogenetic provinces in Nigeria: Economic Geology, v. 68 , no. 2 , p. $278-280$.

Pecora, W. T., 1956, Carbonatites-a review: Geological Society of America Bulletin, v. 67 , no. 11 , p. $1537-1556$.

Permingeat, François, 1957, Le gisement de molybdène, tungstène, et cuivre d'Azegour (Haut Atlas); étude pétrographique et métallogenique: Morocco Service Géologique, Notes et Mémoires no. 141, $284 \mathrm{p}$.

Quan, C. K., 1978, Fluorine: U.S. Bureau of Mines Mineral Commodity Profile 20, 27 p.

Reed, A. H., and Clarke, R. G., 1976, The mineral industry of Kenya, Tanzania, and Uganda: U.S. Bureau of Mines Minerals Yearbook 1973, v. 3, p. 533-545.

Sainfeld, Paul, 1956, The lead-zinc-bearing deposits of Tunisia: Economic Geology, v. 51, no. 2 , p. $150-177$. 
Sainsbury, C. L., 1964, Association of beryllium with tin deposits rich in fluorite: Economic Geology, v. 59, no 5, p. 920-926.

1969, Tin resources of the world: U.S. Geological Survey Bulletin 1301, 55 p.

Shawe, D. R., Van Alstine, R. E., Worl, R. G., Heyl, A. V., Trace, R. D., Parker, R. L., Griffitts, W. R., Sainsbury, C. L., and Cathcart, J. B., 1976, Geology and resources of fluorine in the United States: U.S. Geological Survey Professional Paper 933, 99 p.

Sheppard, R. A., and Gude, A. J., 3d, 1969, Authigenic fluorite in Pliocene lacustrine rocks near Rome, Malheur County, Oregon: U.S. Geological Survey Professional Paper 650-D, p. D69-D74.

1976, Fluorite: U.S. Geological Survey Professional Paper 1000, p. 48.

Smith, W. C., 1956, A review of some problems of African carbonatites [presidential address]: Geological Society of London, Quarterly Journal, v. 112, pt. 2, no. 446, p. 189-219.

South African Mining and Engineering Journal, 1970, Zwartkloof to produce soon: v. 81 (pt. 2), no. 4035 , p. 5-9.

Spada, Aldo, 1969, Il giacimento di fluorite e baritina esalativo-sedimentario in "facies" lacustre, intercalato nei sedimenti piroclastici della zona di Castel Giuliano, in prov. di Roma: Industria Mineraria, v. 20, no. 10, p. 501, 504-510, 515-518.

Stowasser, W. F., 1976, The mineral industry of Morocco: U.S. Bureau of Mines Minerals Yearbook 1973, v. 3, p. 623-632.

Thibieroz, Jacques, 1974, Hammam Djedidi et Hammam Zriba, Région de Zaghouan, Tunisie: Paris, L'Université Pierre et Marie Curie, Ph.D. thesis, 367 p.

Tuttle, O. F., and Gittins, J., eds, 1966, Carbonatites: New York, John Wiley \& Sons, 591 p.

U.S. Bureau of Mines, 1951, Fluorspar, South West Africa: Mineral Trade Notes, v. 33, no. 6 , p. $37-38$. 
-1953, Tunisia: Mineral Trade Notes, v. 36, no. 2, p. 49-50.

1970, Republic of South Africa: Mineral Trade Notes, v. 67, no. 12, p. 22-23.

1972, Tunisia: Mineral Trade Notes, v. 69, no. 8, p. 15-16.

1973, Fluorspar-Mozambique: Mineral Trade Notes, v. 70, no. 12, p. 16-17.

Vail, J. R., 1978, Outline of the geology and mineral deposits of the Democratic Republic of Sudan and adjacent areas: London, Institute of Geological Sciences, Overseas geology and mineral resources, no. 49, $66 \mathrm{p}$.

Van Alstine, R. E., 1976, Continental rifts and lineaments associated with major fluorspar districts: Economic Geology, v. 71, no. 6, p. 977-987.

Van Zijl, P. J., 1962, The geology, structure, and petrology of the alkaline intrusions of Kalkfeld and Okorusu and the invaded Damara rocks: [South Africa] Stellenbosch University Annals, ser. A, v. 37, no. 4, p. 237-346.

Verwoerd, W. J., 1967, The carbonatites of South Africa and South West Africa: South Africa Geological Survey Handbook 6, 452 p.

Von Gruenewaldt, Gerhard, 1977, The mineral resources of the Bushveld Complex: [South Africa] Minerals Science and Engineering, South Africa, v. 9, no. 2, p. 83-95.

Watson, M. D., and Snyman, C. P., 1975, The geology and mineralogy of the fluorite deposits at the Buffalo fluorspar mine on Buffelsfontein, $347 \mathrm{KR}$, Naboomspruit district: Geological Society of South Africa Transactions and Proceedings, v. 78 , pt. 1 , p. $137-151$.

Whiteman, A. J., 1971, The geology of the Sudan Republic: Oxford, Clarendon Press, 290 p.

Williams, F. A., Meehan, J. A., Paulo, K. L., John, T. U., and Rushton, H. G., 1956, Economic geology of the decomposed columbite-bearing granites, Jos Plateau, Nigeria: Economic Geology, v. 51, no. 4, p. 303-332. 


